\title{
Tendência temporal e perfil epidemiológico da mortalidade de idosos em decorrência de quedas em Pernambuco, Brasil, 2001-2015
}

\author{
Temporal trend and epidemiological profile of mortality in the elderly due to falls in \\ Pernambuco, Brazil, 2001-2015
}

Tendencia temporal y perfil epidemiológico de mortalidad en ancianos por caídas en

Pernambuco, Brasil, 2001-2015

\author{
Renato de Souza Mariano1, Thais Silva Matos², Carlos Dornels Freire de Souza ${ }^{3}$ \\ 1- Fisioterapeuta. Instituto de Medicina Integral Professor Fernando Figueira (IMIP). Unidade \\ Pernambucana de Atenção Especializada (UPAE). Petrolina, Pernambuco, Brasil. \\ 2- Fisioterapeuta. Professora da Faculdade São Francisco de Juazeiro (FASJ). Juazeiro, Bahia, Brasil. \\ 3- Professor do Curso de Medicina da Universidade Federal de Alagoas (UFAL), campus Arapiraca, \\ Alagoas, Brasil. \\ DOI: $10.28998 / 2525-4200.2019 v 4 n 3.1281-1292$
}

\section{RESUMO}

Objetivo: descrever a tendência e o perfil epidemiológico da mortalidade de idosos em decorrência de quedas no estado de Pernambuco, Brasil, no período de 2001 a 2015. Métodos: estudo observacional incluindo todos os óbitos de indivíduos com 60 anos ou mais em decorrência de quedas no estado de Pernambuco. Os dados foram obtidos do Sistema de Informações sobre Mortalidade (SIM). Foram analisadas variáveis epidemiológicas (faixa etária, sexo, raça/cor, escolaridade, estado civil, local de ocorrência, categoria do CID-10) e as taxas de mortalidade geral bruta e padronizada pela população mundial, além das taxas específicas segundo sexo e categoria do CID-10. Para as análises, foram utilizados o modelo de regressão por pontos de inflexão e a estatística descritiva. Resultados: foram registrados 2080 óbitos no período estudado, com taxa de mortalidade média de 22,68/100 mil e tendência significativa de crescimento $(4,5 \%$ ao ano). A taxa de mortalidade masculina $(24,3 / 100$ mil) foi superior à feminina $(21,4 / 100$ mil), embora em termos proporcionais, $54,7 \%$ dos óbitos foram de mulheres. Um percentual de $55,5 \%$ dos óbitos tinham 80 ou mais anos, 51,4\% eram pardos e 50,5\% possuíam baixa escolaridade (analfabeto ou até três anos de estudo). 34,6\% dos óbitos foram em decorrência do código W18 (outras quedas do mesmo nível). Conclusão: a mortalidade de idosos em razão de quedas mostrou crescimento no estado de Pernambuco. O perfil observado mostra a importância de políticas públicas voltadas para a prevenção destes eventos.

Palavras-chaves: Acidentes por Quedas; Assistência a Idosos; Mortalidade. 


\begin{abstract}
Objective: to describe the trend and epidemiological profile of mortality in the elderly due to falls in the state of Pernambuco, Brazil, from 2001 to 2015. Methods: observational study including all deaths of individuals aged 60 or over in due to falls in the state of Pernambuco. Data were obtained from the Mortality Information System (SIM). Epidemiological variables (age group, gender, race / color, education, marital status, place of occurrence, category of ICD-10) and the general gross and standardized mortality rates by the world population were analyzed, in addition to specific rates according to sex and category of ICD-10. For the analyzes, the inflection point regression model and descriptive statistics were used. Results: 2080 deaths were registered in the studied period, with an average mortality rate of 22.68 / 100 thousand and a significant growth trend (4.5\% per year). The male mortality rate (24.3 / 100 thousand) was higher than that of women, although in proportional terms, $54.7 \%$ of deaths were women. A percentage of $55.5 \%$ of deaths were 80 years old or more, $51.4 \%$ were brown and $50.5 \%$ had low education (illiterate or up to 3 years of study). $34.6 \%$ of deaths were due to the W18 code (other falls at the same level). Conclusion: the mortality of elderly people due to falls showed an increase in the state of Pernambuco. The observed profile shows the importance of public policies aimed at preventing these events.
\end{abstract}

Keywords: Accidental Falls; Old Age Assistance; Mortality

\title{
RESUMEN
}

Objetivo: describir la tendencia y el perfil epidemiológico de la mortalidad en los ancianos debido a caídas en el estado de Pernambuco, Brasil, de 2001 a 2015. Métodos: estudio observacional que incluye todas las muertes de personas de 60 años o más en debido a caídas en el estado de Pernambuco. Los datos se obtuvieron del Sistema de Información de Mortalidad (SIM). Se analizaron las variables epidemiológicas (grupo de edad, sexo, raza/color, educación, estado civil, lugar de ocurrencia, categoría de ICD-10) y las tasas generales de mortalidad brutas y estandarizadas por la población mundial, además de tasas específicas según el sexo y la categoría. de la CIE-10. Para los análisis, se utilizó el modelo de regresión de puntos de inflexión y estadísticas descriptivas. Resultados: se registraron 2080 muertes en el período estudiado, con una tasa de mortalidad promedio de 22.68/100 mil y una tendencia de crecimiento significativa $(4.5 \%$ por año). La tasa de mortalidad masculina (24.3/100 mil) fue mayor que la de las mujeres, aunque en términos proporcionales, el $54.7 \%$ de las muertes fueron mujeres. Un porcentaje del $55.5 \%$ de las muertes tenía 80 años o más, el $51.4 \%$ era marrón y el $50.5 \%$ tenía poca educación (analfabetos o hasta 3 años de estudio). El 34,6\% de las muertes se debieron al código W18 (otras caídas en el mismo nivel). Conclusión: la mortalidad de las personas mayores por caídas mostró un aumento en el estado de Pernambuco. El perfil observado muestra la importancia de las políticas públicas destinadas a prevenir estos eventos.

Palablas clave: Accidentes por Caídas; Asistencia a los Ancianos; MORTIALIDAD 


\section{INTRODUÇÃO}

O crescente aumento da população idosa é fato conhecido mundialmente. Segundo a Organização Pan-Americana da Saúde - OPAS, o número de idosos com mais de 60 anos chegará a dois bilhões até o ano de $2050^{1}$. Projeções brasileiras apontam que em 2042 , o país terá $24,5 \%$ da população com mais de 60 anos, contabilizando 57 milhões de idosos, quinta maior população idosa do mundo ${ }^{2}$.

Com o envelhecimento, surgem alterações fisiológicas e patológicas decorrentes do declínio funcional, ampliando o risco de doenças cardiovasculares, déficits sensoriais (auditivo e visual) e cognitivos e afecções osteomioarticulares ${ }^{3,4}$. Nesse sentido, o idoso torna-se cada vez mais vulnerável, assim interferindo na funcionalidade, independência e autonomia, bem como no desempenho das atividades básicas do dia-a-dia ${ }^{5}$.

Dentre os eventos que comprometem a funcionalidade e elevam o risco de mortalidade precoce, destacam-se as quedas, um evento frequente nesse segmento populacional e que tem se tornado um problema de saúde pública na atualidade ${ }^{6,7}$. Estima-se que em média 30\% das pessoas com 65 anos já sofreram algum episódio de queda ${ }^{8}$. Em idosos com 70 anos ou mais, esse percentual sobe para $40 \%$, influenciando no aumento da morbidade e da mortalidade em idosos ${ }^{8}$.

A queda é definida como um evento frequente e limitante, que ocorre de forma inesperada e não intencional, resultando na mudança de posicionamento do indivíduo para um nível mais baixo em relação à posição inicial, com incapacidade de correção em tempo hábil ${ }^{7}$. É um resultado da perda do equilíbrio postural e da ineficiência dos mecanismos necessários para a manutenção do controle postural ${ }^{7}$.

No período de 1996 a 2012 ocorreram 941.923 internações e 66.876 óbitos na população idosa em decorrência de quedas no Brasil ${ }^{8}$. Nesse período, a taxa de mortalidade aumentou $200 \%$, passando de 1,25 para 3,75/10.000 idosos, o que caracteriza um crescimento de $15 \%$ ao ano ${ }^{8}$.

Com base no exposto, este trabalho objetivou descrever a tendência temporal e o perfil epidemiológico da mortalidade de idosos em decorrência de quedas no estado de Pernambuco, Brasil, no período de 2001 a 2015.

\section{MÉTODOS}

\section{Desenho, população e período de estudo}


Trata-se de um estudo epidemiológico observacional envolvendo todos os óbitos em decorrência de quedas em idosos (indivíduos com 60 anos ou mais) residentes no estado de Pernambuco no período de 2001 a 2015.

\section{Cenário de estudo}

Pernambuco é um dos nove estado no Nordeste brasileiro, possui uma população estimada para 2019 de 9,5 milhões de habitantes, Índice de Desenvolvimento Humano (IDH 0,673), ocupando a $19^{\text {a }}$ posição dentre os estados brasileiros ${ }^{9}$. Em 2000, apenas $8,9 \%$ da população pernambucana tinha 60 anos ou mais; em 2010, esse percentual alcançou $10,7 \%^{9}$.

\section{Variáveis de estudo}

Neste estudo, foram incluídas variáveis epidemiológicas (faixa etária, sexo, raça/cor, escolaridade, estado civil, local de ocorrência, categoria do CID-10) e as taxas de mortalidade geral bruta e padronizada pela população mundial, além das taxas específicas segundo sexo e categoria do CID-10.

\section{Procedimentos de coleta de dados}

Os dados foram extraídos do Sistema de Informações sobre Mortalidade (SIM), através da plataforma do Departamento de Informática do Sistema Único de Saúde (Datasus) (http://datasus.saude.gov.br/). Para a coleta, foram considerados os códigos W00 a W19 da Classificação Internacional de Doenças (CID-10): W00- queda no mesmo nível envolvendo gelo e neve, W01- queda no mesmo nível por escorregão, tropeção ou passos em falsos (traspés), W02- queda envolvendo patins de rodas ou para gelo, esqui ou pranchas de rodas, W03- outras quedas no mesmo nível por colisão com ou empurrão por outra pessoa, W04- queda, enquanto estava sendo carregado ou apoiado por outra(s) pessoa(s), W05- queda envolvendo uma cadeira de rodas, W06- queda de um leito, W07queda de uma cadeira, W08- queda de outro tipo de mobília, W09- queda envolvendo equipamento de "playground", W10- queda em ou de escadas ou degraus, W11- queda em ou de escadas de mão, W12- queda em ou de um andaime, W13- queda de ou para fora de edifícios ou outras estruturas, W14- queda de árvore, W15- queda de penhasco, W16- mergulho ou pulo na água causando outro traumatismo que não afogamento ou submersão, W17- outras quedas de um nível a outro, W18- outras quedas no mesmo nível, W19- queda sem especificação. Adicionalmente, os dados populacionais necessários para 
o cálculo das taxas de mortalidade foram obtidos do Instituto Brasileiro de Geografia e Estatística (IBGE).

Após a coleta, as taxas foram padronizadas pelo método direto, considerando a população mundial como padrão e as seguintes faixas etárias: 60-64 anos, 65-69 anos, 70-74 anos, 75-79 anos e 80 anos ou mais.

\section{Tratamento estatístico}

Inicialmente, foi analisada a tendência temporal da taxa de mortalidade específica utilizando o modelo de regressão por pontos de inflexão (joinpoint regression model). As tendências foram classificadas em crescente, decrescente ou estacionária. Calculou-se o percentual de variação anual (APC, annual percent change) e o percentual de variação anual médio (AAPC, average anual percent change) com intervalo de confiança de $95 \%$ (IC95\%) e a significância estatística de 5\%. Em seguida, as variáveis epidemiológicas foram submetidas à análise descritiva simples (frequências absolutas e relativas).

As análises foram realizadas com o auxílio dos softwares Joinpoint regression (versão 4.6.0.0) e Statistical Package for the Social Sciences (SPSS-IBM versão 22.0).

\section{Aspectos éticos}

Por utilizar dados secundários de domínio público, dispensou-se a apreciação pelo Comitê de Ética em Pesquisa.

\section{RESULTADOS}

No período de 2001 a 2015, foram registrados 2080 óbitos de idosos em decorrência de quedas no estado de Pernambuco, com taxa de mortalidade bruta igual a 22,68 óbitos/ 100 mil idosos e padronizada igual a 22,33/100 mil. A taxa de mortalidade foi maior na população masculina (24,3/100 mil) do que na feminina (21,40/100 mil). Ao longo da série temporal, as taxas de mortalidade geral (bruta e padronizada) e específicas segundo sexo apresentaram tendência de crescimento: em ambos os sexos, a taxa bruta passou de 18,90/100 mil em 2001 para 33,97/100 mil em 2015 (incremento anual de 4,5\%), a taxa padronizada cresceu de 18,43 para 33,59/100 mil (incremento anual de 4,6\%), a masculina cresceu de 20,54 para 32,52/100 mil (incremento anual de 3,6\%) e a feminina passou de 17,65 para 35,01/100 mil (incremento anual de 5,3\%) (Figura 1). 
Figura 1- Evolução temporal taxa de mortalidade específica de idosos por quedas em Pernambuco, Brasil, 2001-2015.
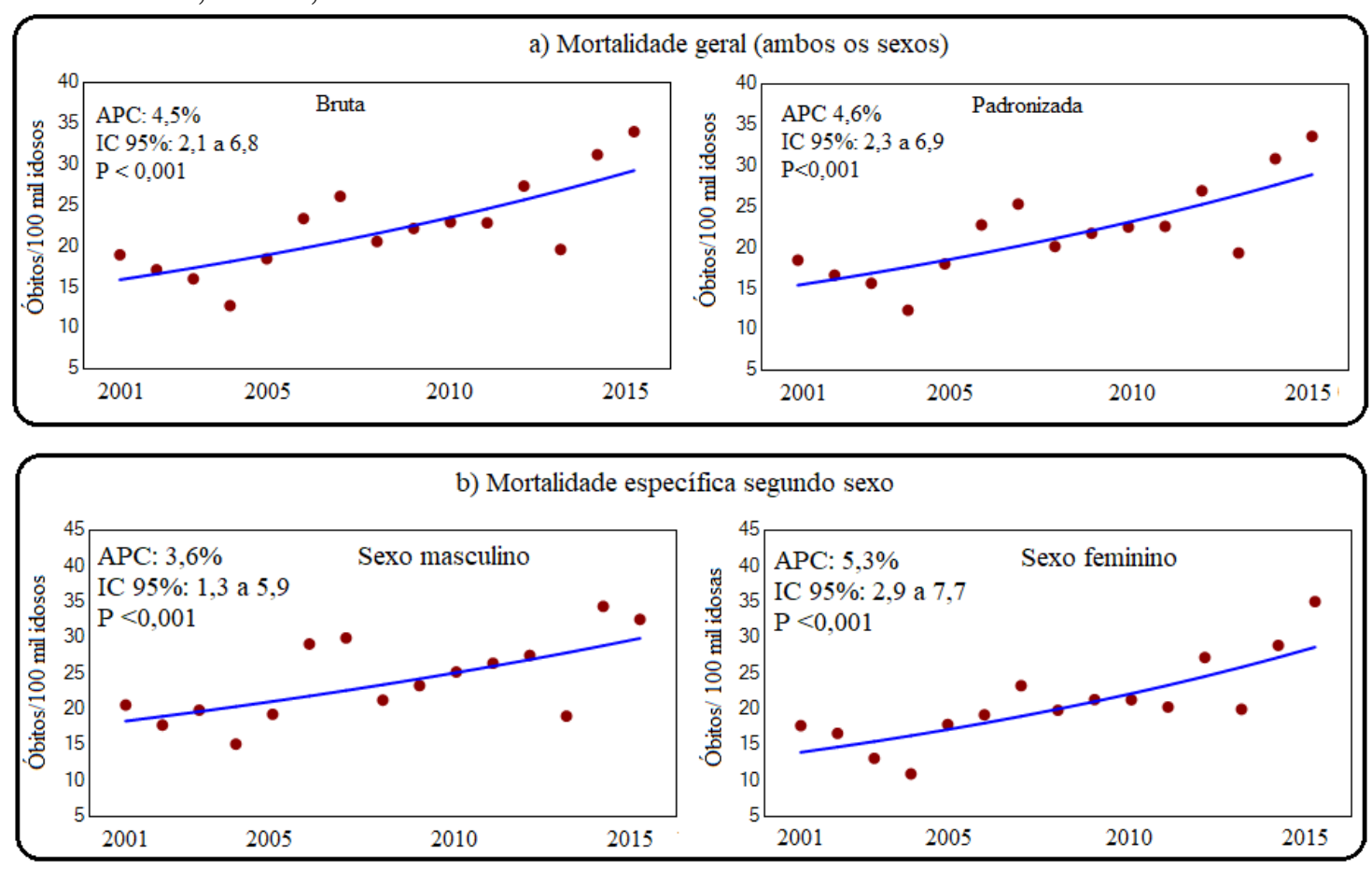

Legenda: APC: Annual percent change (Variação percentual anual); IC 95\%: Intervalo de confiança de $95 \%$.

Um percentual de 55,5\% ( $\mathrm{n}=1554)$ dos óbitos ocorreu em idosos com 80 anos ou mais, 54,7\% $(n=1531)$ eram do sexo feminino, 51,4\% $(n=1440)$ da raça parda, 61,2\% $(\mathrm{n}=1714)$ com até sete anos de estudo, 33,7\% ( $\mathrm{n}=944)$ viúvos e 81,7\% ( $\mathrm{n}=2288)$ dos óbitos ocorreram no ambiente hospitalar (Tabela 1).

Tabela 1- Caracterização epidemiológica dos óbitos de idosos em decorrência de quedas em Pernambuco, Brasil, 2001-2015.

\begin{tabular}{|c|c|c|}
\hline Variável & & \\
\hline \multicolumn{3}{|l|}{ Faixa etária } \\
\hline 60 a 69 anos & 493 & 17,6 \\
\hline 70 a 79 anos & 754 & 26,9 \\
\hline 80 anos ou mais & 1554 & 55,5 \\
\hline \multicolumn{3}{|l|}{ Sexo } \\
\hline Masculino & 1269 & 45,3 \\
\hline Feminino & 1531 & 54,7 \\
\hline Ignorado & 1 & $<0,0$ \\
\hline \multicolumn{3}{|l|}{ Raça/cor } \\
\hline Branca & 1193 & 42,6 \\
\hline Preta & 59 & 2,1 \\
\hline
\end{tabular}




\begin{tabular}{ccc}
\hline Amarela & 5 & 0,2 \\
Parda & 1440 & 51,4 \\
Ignorado & 104 & 3,7 \\
\hline Escolaridade & & \\
\hline Nenhuma & 799 & 28,5 \\
1 a 3 anos & 616 & 22,0 \\
4 a 7 anos & 299 & 10,7 \\
8 a 11 anos & 168 & 6,0 \\
12 anos e mais & 95 & 3,4 \\
Ignorado & 824 & 29,4 \\
\hline Estado Civil & & \\
\hline Solteiro & 703 & 25,1 \\
Casado & 871 & 31,1 \\
Viúvo & 944 & 33,7 \\
Separado judicialmente & 60 & 2,1 \\
Outro & 20 & 0,7 \\
Ignorado & 203 & 7,3 \\
\hline Local de ocorrência & & \\
\hline Hospital & 2288 & 81,7 \\
Outro estabelecimento de saúde & 49 & 1,7 \\
Domicílio & 369 & 13,2 \\
Via pública & 47 & 1,7 \\
Outros & 44 & 1,6 \\
Ignorado & 4 & 0,1 \\
\hline Total & $\mathbf{2 8 0 1}$ & $\mathbf{1 0 0 , 0}$ \\
\hline & &
\end{tabular}

Ao estratificar os óbitos segundo categoria do CID-10, destacou-se a mortalidade pelo código W18 (Outras quedas do mesmo nível), correspondendo a 34,6\% dos óbitos ( $n=969)$ e taxa de mortalidade de 7,85/100 mil. Salienta-se que as mortes por queda sem especificação correspondeu a 44,3\% (n=1240) dos registros (Tabela 2).

Tabela 2- Número de óbitos e taxa de mortalidade específica de idosos por quedas, segundo CID-10, em Pernambuco, Brasil, 2001-2015.

\begin{tabular}{lccc}
\hline \multicolumn{1}{c}{ Categoria CID-10 } & $\mathbf{N}^{\mathbf{0}}$ óbitos & $\mathbf{\%}$ & TME/100 mil \\
\hline $\begin{array}{l}\text { W01- queda no mesmo nível por escorregão, tropeção ou } \\
\text { passos em falsos (traspés) }\end{array}$ & 145 & 5,2 & 1,17 \\
$\begin{array}{l}\text { W02- queda envolvendo patins de rodas ou para gelo, esqui } \\
\text { ou pranchas de rodas }\end{array}$ & 2 & 0,1 & 0,02 \\
$\begin{array}{l}\text { W03- outras quedas no mesmo nível por colisão com ou } \\
\text { empurrão por outra pessoa }\end{array}$ & 1 & 0,0 & 0,01 \\
W05- queda envolvendo uma cadeira de rodas & 3 & 0,1 & 0,02 \\
W06- queda de um leito & 114 & 4,1 & 0,92 \\
W07- queda de uma cadeira & 36 & 1,3 & 0,29 \\
W08- queda de outro tipo de mobília & 8 & 0,3 & 0,06
\end{tabular}




\begin{tabular}{lccc} 
W09- queda envolvendo equipamento de "playground" & 1 & 0,0 & 0,01 \\
W10- queda em ou de escadas ou degraus & 75 & 2,7 & 0,61 \\
W11- queda em ou de escadas de mão & 12 & 0,4 & 0,10 \\
W12- queda em ou de um andaime & 8 & 0,3 & 0,06 \\
W13- queda de ou para fora de edifícios ou outras estruturas & 83 & 3,0 & 0,67 \\
W14- queda de árvore & 21 & 0,7 & 0,17 \\
W15- queda de penhasco & 7 & 0,2 & 0,06 \\
W16- mergulho ou pulo na água causando outro traumatismo & & & 0,02 \\
que não afogamento ou submersão & 2 & 0,1 & 0,60 \\
W17- outras quedas de um nível a outro & 74 & 2,6 & 7,85 \\
W18- outras quedas no mesmo nível & 969 & 34,6 & 10,04 \\
W19- queda sem especificação & 1240 & 44,3 & $\mathbf{2 2 . 6 8}$ \\
\hline \multicolumn{1}{c}{ Total } & $\mathbf{2 8 0 1}$ & $\mathbf{1 0 0 . 0}$ & \\
\hline
\end{tabular}

Legenda: TME- Taxa de Mortalidade Específica; Não foram registrados casos para os CIDs W00- queda no mesmo nível envolvendo gelo e neve e W04- queda, enquanto estava sendo carregado ou apoiado por outra(s) pessoa(s).

\section{DISCUSSÃO}

Neste estudo, a taxa de mortalidade média em homens foi maior do que a observada em mulheres, embora a tendência de crescimento percentual anual seja maior na população feminina. O perfil foi caracterizado por idosos com mais de 80 anos, predomínio de óbitos femininos, baixa escolaridade, raça parda, viúvos e com óbito ocorrendo em unidade hospitalar. Quedas de um mesmo nível foram a principal causa de óbitos.

O comportamento temporal de crescimento da mortalidade segue o padrão mundial e nacional ${ }^{11-13}$. No Brasil, a taxa de óbitos em idosos entre 1997 e 2010 superou 30/100.00 habitantes, e $0,61 \%$ dos óbitos foram ocasionados em decorrência de quedas 12. A taxa de mortalidade de idosos em decorrência de quedas nas capitais brasileiras aumentou 200\% entre 1996 e 2012, passando de 1,25 para 3,75/10.000 idosos com incremento de $15 \%$ ao ano ${ }^{14}$.

Estudos realizados em Amparo - São Paulo e Pelotas - Minas Gerais detectaram que $21,5 \%$ e $43,4 \%$ dos idosos avaliados apresentavam síndrome da fragilidade, respectivamente ${ }^{15-17}$. Esta é uma condição associada ao envelhecimento, podendo ser precursora de incapacidade e quedas decorrente da diminuição de massa e da força muscular, exaustão, alteração da marcha e do equilíbrio, tonturas, vertigens e alteração da densidade mineral óssea ${ }^{15-17}$. Quanto mais idoso, maior a fragilidade apresentada, 
culminado em um maior risco de quedas e, consequentemente, hospitalização e morte ${ }^{15-}$ 17.

Além disso, fatores como função neuromuscular prejudicada, história prévia de quedas, prejuízos psicocognitivos, polifarmácia, uso de benzodiazepínicos, presença de ambiente físico inadequado, incapacidade funcional e hipotensão postural conspiram para um risco maior de quedas na população idosa ${ }^{18,19}$. Uma vez identificados os fatores de risco de maneira precoce, as quedas pode ser potencialmente evitadas ${ }^{18,19}$.

As taxas de mortalidade em nosso estudo foram mais elevadas para o sexo masculino em todo o período analisado. O Brasil, o Distrito Federal e mais 20 capitais federais apresentaram tendência de aumento nas taxas de mortalidade para o sexo masculino e feminino, segundo estudo de Abreu e colaboradores ${ }^{14}$. As quedas no sexo masculino estão associadas ao maior envolvimento do homem em atividades físicas intensas e perigosas, ignorando os limites de sua capacidade física ${ }^{14-16}$.

Adicionalmente, estudo envolvendo 1444 idosos em atendimento pré-hospitalar vítimas de queda em Maringá/ Paraná, entre 2006 e 2008, mostrou que a gravidade do traumatismo em homens é maior que em mulheres ${ }^{20}$. Investigação realizada na Bahia, com dados originados do Sistema de Informação Hospital do Sistema Único de SaúdeSIH/SUS no ano de 2014, revelou que 251 óbitos foram registrados em idosos internados por quedas, sendo que a maior proporção de mortes foi entre os homens $(55,4 \%)^{21}$, dados que corroboram os nossos achados.

Estudo realizado em Natal/Rio Grande do Norte, observou predomínio da faixa etária com maior mortalidade foi de 80 anos ou mais $(62,86 \%)$ e raça/cor parda $(58,62 \%)$. Em relação à escolaridade, o percentual de destaque foi para os que não tinham nenhuma $(36,36 \%)$, e 30,30\% tinham de um a três anos de estudo. Já, quanto ao estado civil, ficou evidenciado que $41,54 \%$ eram casados, e $29,23 \%$, solteiros ou viúvos ${ }^{22}$. Esse perfil tem sido observado na maioria dos estudos brasileiros e está em consonância com os achados observados em Pernambuco.

Corroborando com os nossos achados, o inquérito para vigilância dos acidentes e violências (VIVA), realizado em Natal/Rio Grande do Norte, apontou que as quedas em geral ocuparam o percentual de $26,7 \%$ das ocorrências registradas; quanto aos tipos de quedas, as de mesmo nível representaram 50,3\% dos registros, quanto às quedas de outros níveis, destaca-se o percentual de $13,2 \%{ }^{23}$. Adicionalmente, estudo realizado em São Paulo apontou que as quedas no mesmo nível foram responsáveis pela maior proporção de mortes definidas (35\%). Salientamos a importância do desenvolvimento de ações que 
possam reduzir essas ocorrências, tendo em vista que, na sua maioria, ocorre dentro do próprio lar ou nas imediações. Nesse sentido, tem destaque a importância da atenção primária à saúde que, através da Estratégia Saúde da Família (ESF) e Núcleo de Apoio à Saúde da Família (NASF), deve conhecer o perfil sociodemográfico local e desenvolver estratégias voltadas para as necessidades singulares dos diferentes grupos etários ${ }^{25}$.

Dentre as limitações deste estudo, destacamos a elevada proporção de óbitos cuja natureza da queda não tenha sido especificada (44,3\%). Percentual semelhante ao observado em Natal/RN, cuja proporção alcançou $48 \%{ }^{24}$. A falta de detalhamento das informações no Sistema de Informação sobre mortalidade representa uma baixa qualidade dos dados utilizados ${ }^{24}$, representando uma limitação importante deste estudo. Melhorias na qualidade dos registros, investigação de óbitos e adequado preenchimento do documento podem contribuir para uma melhor clareza da etiologia das quedas e, desse modo, permitir o desenvolvimento de ações que possam evitar essas ocorrências.

\section{CONCLUSÃO}

No período estudado, observou-se crescimento da taxa de mortalidade de idosos em decorrência de quedas no estado de Pernambuco, sendo a taxa masculina superior à observada na população feminina, embora o percentual de crescimento anual seja maior nesse grupo populacional. O perfil epidemiológico foi caracterizado pelo predomínio de mulheres, faixa etária com 80 anos ou mais, raça parda, baixa escolaridade e o desfecho óbito ocorrendo no hospital. Houve predomínio de quedas do mesmo nível.

A tendência temporal e o perfil epidemiológico predominante sinalizam a gravidade do problema no estado. Políticas, planos e/ou estratégias devem ser desenvolvidas a fim de reduzir a ocorrência de quedas em idosos, evitando, deste modo, a ocorrência do desfecho óbito.

\section{REFERÊNCIAS}

1- Organização das Nações Unidas (ONU): Relatório sobre envelhecimento alerta para 2050, ONU, 2009. [cited 2020 jan 15]. Available from https://nacoesunidas.org/acao/pessoas-idosas/. 
2- Organização Pan-Americana da Saúde (OPAS), Brasília, 2018. [cited 2020 jan 15]. Available from https://www.paho.org/bra/index.php?option=com_content\&view $=$ article\&id=5661:folhainformativa-envelhecimento-e-saude $\&$ Itemid $=820$.

3- Silva SLA, Vieira RA, Arante SP, Dias RC. Avaliação de fragilidade, funcionalidade e medo de cair em idosos atendidos em um serviço ambulatorial de geriatria e gerontologia. Fisioterapia e Pesquisa. 2009;16(2):120-125.

4- Matos FS, Jesus CSD, Carneiro JAO, Coqueiro RDS, Fernandes MH, Brito TA. Redução da capacidade funcional de idosos residentes em comunidade: estudo longitudinal. Cien Saúde Colet. 2018; (23):3393-401.

5- Souza CDF, Fernandes TRM, Matos TS, Ribeiro Filho JM, Almeida GKA, Lima JCB et al. Grau de incapacidade física na população idosa afetada pela hanseníase no estado da Bahia, Brasil. Acta fisiatrica [Internet]. 2017;24(1):27-2.

6- FECHINE, BRA; TROMPIERI, N. O processo de envelhecimento: as principais alterações que acontecem com o idoso com o passar dos anos. Revista científica internacional. 2012; 20(1):1-7.

7- Tomicki C, Cecagno Zanini SC,Cecchin L, et al. Efeito de um programa de exercícios físicos no equilíbrio e risco de quedas em idosos institucionalizados: ensaio clínico randomizado. Rev. Bras. Geriatr. Gerontol. 2016;19(3):473-482.

8- World Health Organization (WHO). Falls. Fact sheet $\mathrm{N}^{\circ}$ 344. [Internet]. 2012 [cited 2015 Mar 07]; Available from: http://www.who.int/mediacentre/factsheets/fs344 /en/

9- Abreu DRDOM, Novaes ES, Oliveira RRD, Mathias TADF, Marcon SS. Internação e mortalidade por quedas em idosos no Brasil: análise de tendência. Cien Saúde Colet. 2018;(23):1131-141.

10- Instituto Brasileiro de Geografia e Estatística (IBGE). Estimativa poulacionais [Internet]. Rio de Janeiro: IBGE; 2019 [cited 2020 jan 15]. Available from: https://cidades.ibge.gov.br/brasil/pe

11- Lagarto S, Nunes C, Gomes D, Mendes MF. A evolução na mortalidade da população idosa portuguesa, por sexos e por causas de morte: Identificação de aglomerados espácio-temporais por NUTSIII. RED. 2013 (1):5-30.

12- Antes DL, Schneider IJC, d'Orsi E. Mortality caused by accidental falls among the elderly: a time series analysis. Rev. Bras. Geriatr. Gerontol. 2015; 18 (4): 769-78.

13- Bacurau AGDM, Ferraz RDO, Donalisio MR, Francisco PMSB. Mortalidade por doenças cerebrovasculares em idosos e a vacinação contra a influenza: Estado de São Paulo, Brasil, 1980-2012. Cad Publica. 2019; 35: e00145117. 14- Abreu DRDOM, Novaes ES, Oliveira RRD, Mathias TADF, Marcon SS. Internação e mortalidade por quedas em idosos no Brasil: análise de tendência. Cien Saúde Colet. 2018;(23):1131-141. 
15- Carmo LV, Drummond LP, Arantes PMM. Avaliação do nível de fragilidade em idosos participantes de um grupo de convivência. Fisioterapia e Pesquisa. 2011; 18 (1):17-22.

16- Augusti ACV, Falsarella GR, Coimbra AMV. Análise da síndrome da fragilidade em idosos na atenção primária-Estudo transversal Rev Bras Med Fam Comunidade. 2017;12(39):1-9.

17- Liano PMPD, Lange C, Sequeira CADC, Jardim VMDR, Castro DSP, Santos F. Fatores associados à síndrome da fragilidade em idosos rurais. Rev Bras Enferm. 2019; $72: 14-21$.

18- Maciel A. Quedas em idosos: um problema de saúde pública desconhecido pela comunidade e negligenciado por muitos profissionais da saúde e por autoridades sanitárias brasileiras. Rev Méd Minas Gerais. 2010; 20(4): 554-57.

19- Siqueira FV, Facchini LA, Piccini RX, Tomasi E, Thumé E, Silveira DS, et al . Prevalência de quedas em idosos e fatores associados. Rev Saúde Pública. 2007;41(5):749-56.

20- MESCHIAL WC, Soares DFPP, Oliveira NLB, Nespollo AM, Silva WA, Santil FLP. Idosos vítimas de quedas atendidos por serviços pré-hospitalares: diferenças de gênero. Rev. bras. epidemiol. 2014; 17(1):3-16.

21- Andrade IR, Souza EA, Luz LA, Pinto Júnior EP. Características e gastos com hospitalizações por quedas em idosos na Bahia. J Health Sci Inst. 2017;35(1):28-31

22- Araújo AM. Perfil da mortalidade por quedas em idosos. Perfil da mortalidade por quedas em idosos. J. res.: fundam. care. online. 2014; 6(3): 863-75.

23- Brasil. Ministério da Saúde. Viva: vigilância de violências e acidentes, 2008 e 2009. Brasília: Ministério da Saúde; 2010.

24- Gawryszewski, Vilma Pinheiro. A importância das quedas no mesmo nível entre idosos no estado de São Paulo. Rev Assoc Med Bras. 2010;56(2):162-67, 2010.

25- Sousa CDF. Programa Saúde da Família - PSF: a ação do estado, o cenário de implantação e a importância do território para a sua efetivação. Hygeia. 2014; 10(18): 207-21. 